\title{
Extending stochastic ordering to belief functions on the real line
}

\author{
Thierry Denœux \\ HEUDIASYC, UTC, CNRS \\ Centre de Recherche de Royallieu \\ BP 20529, F-60205 Compiègne, France
}

January 7, 2009 


\begin{abstract}
In this paper, the concept of stochastic ordering is extended to belief functions on the real line defined by random closed intervals. In this context, the usual stochastic ordering is shown to break down into four distinct ordering relations, called credal orderings, which correspond to the four basic ordering structures between intervals. These orderings are characterized in terms of lower and upper expectations. We then derive the expressions of the least committed (least informative) belief function credally less (respectively, greater) than or equal to a given belief function. In each case, the solution is a consonant belief function that can be described by a possibility distribution. A simple application to reliability analysis is used as an example throughout the paper.

Keywords: Dempster-Shafer theory, Evidence theory, Random intervals, Ordering relation, Possibility distribution.
\end{abstract}




\section{Introduction}

Stochasting ordering is an important concept in Probability theory. Given two probability distributions $P$ and $P^{\prime}$ on the real line, we say that $P$ is stochastically less than or equal to $P^{\prime}$ and we write $P \leq_{\text {st }} P^{\prime}$ if

$$
P((x,+\infty)) \leq P^{\prime}((x,+\infty)), \quad \forall x \in \mathbb{R}
$$

or, equivalently,

$$
F(x) \geq F^{\prime}(x), \quad \forall x \in \mathbb{R},
$$

where $F$ and $F^{\prime}$ are the cumulative distribution functions (cdfs) of $P$ and $P^{\prime}$, respectively [33]. Intuitively, this means that distribution $P$ attaches less probability to larger values than $P^{\prime}$ does. It is well known that (1) holds if and only if:

$$
\int g \mathrm{~d} P \leq \int g \mathrm{~d} P^{\prime}
$$

for all non decreasing real function $g$ for which the expectations (integrals) are defined [21]. The concept of stochastic ordering has numerous applications in hypothesis testing, queuing theory, reliability, decision theory, etc.

In recent years, the theory of belief functions, or Dempster-Shafer theory has been gaining increasing attention as a generalization of probability theory allowing the representation of various states of knowledge ranging from complete certainty to outright ignorance $[6,26,30,37]$. This formalism has been applied in various areas including statistics [8, 19], classification [11, 24, 4], clustering [10, 22], data mining [34], decision analysis [31], knowledge elicitation [17], etc. Roughly speaking, a belief function on a domain $\Omega$ is based on a mass function assigning numbers in the $[0,1]$ interval to subsets of $\Omega$, called focal sets. When the domain is the real line, focal sets are usually constrained to be real intervals $[7,29]$. A belief function is then mathematically equivalent to a random interval.

In this paper, we extend the concept of stochastic ordering to belief functions on the real line. We show that, in this context, the usual stochastic ordering breaks down into four distinct orderings, derived from the four basic ordering structures between intervals 1. These orderings, called credal orderings, can be characterized in terms of lower and upper expectations. We derive the expressions of the least committed (least informative) belief function credally less (respectively, greater) than or equal to a given belief function. A running example related to reliability analysis is used as an illustration throughout the paper.

The rest of this paper is organized as follows. Background notions on belief functions are recalled in Section 2. Credal orderings are then introduced in Section 3. The expressions of the least committed belief functions subject to credal ordering constraints are then derived in Section 4. Finally, Section 5 concludes the paper.

\section{Belief Functions on the Real Line}

In this paper, basic definitions and results related to belief functions will be assumed to be known. The reader is invited to refer to $[26,30,28]$ for extensive presentations of

\footnotetext{
${ }^{1}$ Some of these orderings were introduced, without development, in [2] and [3] in the context of novelty detection.
} 
these notions. In this section, we will focus on belief functions on the real line that are mathematically defined as random intervals, as introduced by Dempster in [7] and studied by Yager [36], Ferson et al. [15], Petitrenaud and Denœux [23] and Smets [29], among others.

\subsection{Belief Function Induced by a Random Interval}

Let $U$ and $V$ be random variables such that $U \leq V$ with probability one, and let $\Gamma$ be the multi-valued mapping that maps each point $(u, v) \in \mathbb{R}^{2}$ such that $u \leq v$ to the closed interval $[u, v]$. This setting defines a random interval $[U, V]=\Gamma(U, V)$, as well as belief and plausibility functions on $\mathbb{R}$ defined, respectively, by

$$
\begin{aligned}
\operatorname{bel}(A) & =\operatorname{Pr}([U, V] \subseteq A) \\
\operatorname{pl}(A) & =\operatorname{Pr}([U, V] \cap A \neq \emptyset)
\end{aligned}
$$

for all element $A$ of the Borel sigma-algebra $\mathcal{B}(\mathbb{R})$ on the real line [7]. We note that $p l(A)=1-\operatorname{bel}(\bar{A})$, where $\bar{A}$ is the complement of $A$, and bel $\leq p l$. As noted in [7], the knowledge of the values taken by bel for closed intervals is sufficient to recover the joint distribution of $U$ and $V$. Furthermore, the marginal distributions of $U$ and $V$ may be recovered from the values bel and $p l$ for all intervals of the form $(-\infty, x]$. Indeed, the following equalities hold for all $x \in \mathbb{R}$ :

$$
\begin{aligned}
\operatorname{bel}((-\infty, x]) & =\operatorname{Pr}([U, V] \subseteq(-\infty, x])=\operatorname{Pr}(V \leq x)=F_{V}(x) \\
p l((-\infty, x]) & =\operatorname{Pr}([U, V] \cap(-\infty, x] \neq \emptyset)=\operatorname{Pr}(U \leq x)=F_{U}(x) .
\end{aligned}
$$

Let $P_{U}$ and $P_{V}$ denote the probability distributions of $U$ and $V$, respectively. It is clear from (6)-(7) that $P_{U} \leq_{\text {st }} P_{V}$, which will be noted $U \leq_{\text {st }} V$.

Although the joint probability distribution of $U$ and $V$ can be arbitrary, we will focus on two special cases of practical interest, where this distribution is either discrete with a finite number of outcomes, or absolutely continuous.

Let us first consider the discrete case where $\operatorname{Pr}\left(U=u_{i} ; V=v_{i}\right)=m_{i}$ for $i \in\{1, \ldots, n\}$ with $\sum_{i=1}^{n} m_{i}=1$. Let $I_{i}=\left[u_{i}, v_{i}\right]$, and let $m$ be the mass function from the set $\mathcal{I}$ of closed real intervals to $[0,1]$ such that

$$
m\left(I_{i}\right)=m_{i}
$$

for all $i \in\{1, \ldots, n\}$ and $m(I)=0$ for all other $I \in \mathcal{I}$. The belief and plausibility functions are then given by:

$$
\begin{aligned}
\operatorname{bel}(A) & =\sum_{I_{i} \subseteq A} m_{i}, \\
\operatorname{pl}(A) & =\sum_{I_{i} \cap A \neq \emptyset} m_{i},
\end{aligned}
$$

for all $A \in \mathcal{B}(\mathbb{R})$. The intervals $I_{i}$ are called the focal intervals of $m$. Note that this framework can be slightly extended to allow the focal intervals to be open or half-open, with possibly infinite lower or upper bounds [7]. It can also be extended to allow a mass to be assigned to the empty set, resulting in an unnormalized mass function [29]. However, these extensions will not be considered in this paper. 
When the joint probability distribution of $U$ and $V$ is absolutely continuous with density function $f$, then we may define a mass density function (or continuous mass function) $m$ from $\mathcal{I}$ to $\mathbb{R}_{+}$as

$$
m([u, v])=f(u, v), \quad \forall u \leq v .
$$

The following equalities then hold:

$$
\begin{aligned}
\operatorname{bel}(A) & =\iint_{[u, v] \subseteq A} f(u, v) \mathrm{d} u \mathrm{~d} v, \\
p l(A) & =\iint_{[u, v] \cap A \neq \emptyset} f(u, v) \mathrm{d} u \mathrm{~d} v,
\end{aligned}
$$

for all $A \in \mathcal{B}(\mathbb{R})$. Here again, the intervals $[u, v]$ such that $m([u, v])>0$ are called focal intervals of $m$.

Three special cases are of interest:

1. When focal intervals are reduced to points, then $b e l=p l$ is a probability measure, sometimes called in this context a Bayesian belief function.

2. When the focal intervals are nested, $m$ is said to be consonant; the associated plausibility function $p l$ is then a possibility measure [38, 14]. If we denote $\pi(x)=p l(\{x\})$ for all $x \in \mathbb{R}$, we then have

$$
p l(A)=\sup _{x \in A} \pi(x), \quad \forall A \in \mathcal{B}(\mathbb{R}) .
$$

Example 1 Let us consider a piece of equipment that fails on demand according to a Bernouilli process with probability $p$, and let $X$ denote the random variable taking the value 1 if the piece of equipment fails, and 0 otherwise. Assume that an expert is asked to provide his opinion about $p$. Using the so-called method of consonant intervals [1, page 89], he may choose a series of $n$ intervals $\left[u_{i}, v_{i}\right]$ along with values $\gamma_{i}$ such that $\operatorname{bel}\left(\left[u_{i}, v_{i}\right]\right)=\gamma_{i}$, and for $i<j, \gamma_{i}<\gamma_{j}$ and $\left[u_{i}, v_{i}\right] \subset\left[u_{j}, v_{j}\right]$. These nested intervals are the focal sets of a discrete mass function $m_{E}$. The mass assigned to the focal set $\left[u_{i}, v_{i}\right]$ is $m_{i}=\gamma_{i}-\gamma_{i-1}$, with $\gamma_{0}=0$.

Let us now assume that, instead of eliciting expert opinion, we have made $n$ independent observations $X_{1}, \ldots, X_{n}$ of $X$, in which the piece of equipment has been found to fail $r$ times out of $n$. Based on this data, we would like to express our opinion on $p$ in the form of a mass function. A solution to this statistical inference problem was provided by Dempster $[5,7]$. This solution takes the form of the continuous mass function:

$$
\begin{array}{ll}
\left.m([u, v])=\frac{n !}{(r-1) !(n-r-1) !} u^{r-1}(1-v)^{n-r-1}\right) & \text { if } 0<r<n, \\
m([0, v])=n(1-v)^{n-1} & \text { if } r=0, \\
m([u, 1])=n u^{n-1} & \text { if } r=n .
\end{array}
$$

Let $[U, V]$ denote the corresponding random interval. If $r=0, U$ is constant and equal to zero. If $r>0$, the probability density of $U$ is

$$
f_{U}(u)=\int_{u}^{1} f(u, v) \mathrm{d} v=\frac{n !}{(r-1) !(n-r) !} u^{r-1}(1-u)^{n-r},
$$


which is the density of a beta distribution with parameters $r$ and $n-r+1$. Similarly, $V$ is constant and equal to 1 if $r=n$. If $r<n$, its probability density is

$$
f_{V}(v)=\int_{0}^{v} f(u, v) \mathrm{d} u=\frac{n !}{r !(n-r-1) !} v^{r}(1-v)^{n-r-1},
$$

which is the density function of the beta distribution with parameters $r+1$ and $n-r$. Using (7) and (6), we may easily derive the expressions of $\operatorname{bel}((-\infty, p])$ and $p l((-\infty, p])$. If $r<n$, we have

$$
\operatorname{bel}((-\infty, p])=F_{V}(p)=\frac{n !}{r !(n-r-1) !} \int_{0}^{p} v^{r}(1-v)^{n-r-1} \mathrm{~d} v,
$$

which is the incomplete beta function ratio $I_{p}(r+1, n-r)$ [20, page 8]. Using standard formula [20, page 63], it can be computed as:

$$
\operatorname{bel}((-\infty, p])=\sum_{i=r+1}^{n}\left(\begin{array}{c}
n \\
i
\end{array}\right) p^{i}(1-p)^{n-i}
$$

Similarly, we have $p l((-\infty, p])=F_{U}(p)$ and, for $r>0$ :

$$
\begin{aligned}
p l((-\infty, p]) & =\frac{n !}{(r-1) !(n-r) !} \int_{0}^{p} u^{r-1}(1-u)^{n-r} \mathrm{~d} u \\
& =\sum_{i=r}^{n}\left(\begin{array}{c}
n \\
i
\end{array}\right) p^{i}(1-p)^{n-i} .
\end{aligned}
$$

\subsection{Lower and Upper Expectations}

Given a mass function $m$ and the associated belief and plausibility functions bel and $p l$, let $\mathcal{P}$ be the set of probability measures $P$ such that

$$
\operatorname{bel}(A) \leq P(A) \leq p l(A), \quad \forall A \in \mathcal{B}(\mathbb{R}) .
$$

Any $P \in \mathcal{P}$ is said to be compatible with bel. The lower and upper expectations of a bounded and measurable function $g: \mathbb{R} \rightarrow \mathbb{R}$ with respect to $m$ are defined as:

$$
\begin{aligned}
& \underline{\mathbb{E}}_{m}(g)=\inf _{P \in \mathcal{P}} \mathbb{E}_{P}(g) \\
& \overline{\mathbb{E}}_{m}(g)=\sup _{P \in \mathcal{P}} \mathbb{E}_{P}(g),
\end{aligned}
$$

where $\mathbb{E}_{P}(g)=\int g \mathrm{~d} P$ denotes the expectation of $g$ with respect to $P$. In the discrete case, these quantities may be shown [6] to be equal to:

$$
\begin{aligned}
& \underline{\mathbb{E}}_{m}(g)=\sum_{i=1}^{n} m_{i} \inf _{x \in I_{i}} g(x) \\
& \overline{\mathbb{E}}_{m}(g)=\sum_{i=1}^{n} m_{i} \sup _{x \in I_{i}} g(x) .
\end{aligned}
$$


In the continuous case, we have [32]:

$$
\begin{aligned}
& \underline{\mathbb{E}}_{m}(g)=\int_{-\infty}^{+\infty} \int_{u}^{+\infty} f(u, v) \inf _{u \leq x \leq v} g(x) \mathrm{d} v \mathrm{~d} u \\
& \overline{\mathbb{E}}_{m}(g)=\int_{-\infty}^{+\infty} \int_{u}^{+\infty} f(u, v) \sup _{u \leq x \leq v} g(x) \mathrm{d} v \mathrm{~d} u .
\end{aligned}
$$

It is obvious that lower and upper expectations boil down to usual expectation when bel is Bayesian.

Finally, the following proposition holds.

Proposition 1 Let $g$ be a bounded, measurable and nondecreasing function, and let $m$ be a discrete or continuous mass function on $\mathbb{R}$ corresponding to the random set $[U, V]$. Then

$$
\begin{aligned}
& \underline{\underline{\mathbb{E}}}_{m}(g)=\mathbb{E}_{U}(g), \\
& \overline{\mathbb{E}}_{m}(g)=\mathbb{E}_{V}(g),
\end{aligned}
$$

where $\mathbb{E}_{U}(\cdot)$ and $\mathbb{E}_{V}(\cdot)$ denote the expectation with respect to the marginal probability distribution of $U$ and $V$, respectively.

Proof. In the discrete case, we have

$$
\underline{\mathbb{E}}_{m}(g)=\sum_{i=1}^{n} m_{i} \inf _{x \in I_{i}} g(x)=\sum_{i=1}^{n} m_{i} g\left(u_{i}\right)=\mathbb{E}_{U}(g) .
$$

In the continuous case,

$$
\begin{aligned}
\underline{\mathbb{E}}_{m}(g) & =\int_{-\infty}^{+\infty} \int_{u}^{+\infty} f(u, v) \inf _{u \leq x \leq v} g(x) \mathrm{d} v \mathrm{~d} u \\
& =\int_{-\infty}^{+\infty} g(u)\left(\int_{u}^{+\infty} f(u, v) \mathrm{d} v\right) \mathrm{d} u \\
& =\int_{-\infty}^{+\infty} g(u) f_{U}(u) \mathrm{d} u,
\end{aligned}
$$

where $f_{U}(u)$ is the marginal probability density of $U$. The proof for the upper expectation is similar.

Example 2 Continuing Example 1, assume that we want to assess our belief that the piece of equipment will fail. The degree of belief and the plausibility of that event can be shown [1, pp. 250-252] to be equal to:

$$
\operatorname{bel}(X=1)=\underline{\mathbb{E}}_{m}\left(g_{i d}\right), \quad p l(X=1)=\overline{\mathbb{E}}_{m}\left(g_{i d}\right),
$$

where $m$ is the mass function representing our state of information regarding parameter $p$, and $g_{i d}: \mathbb{R} \rightarrow \mathbb{R}$ is the bounded and measurable function defined by

$$
g_{i d}(x)= \begin{cases}x & \text { if } 0 \leq x \leq 1 \\ 0 & \text { otherwise }\end{cases}
$$


If $m$ is a discrete mass function $m_{E}$ as described in Example 1, we thus have

$$
\begin{aligned}
\operatorname{bel}(X=1) & =\sum_{i=1}^{n} m_{i} u_{i} \\
p l(X=1) & =\sum_{i=1}^{n} m_{i} v_{i} .
\end{aligned}
$$

If $m$ is the continuous mass function given by (12)-(14) and $r>0$, then $\operatorname{bel}(X=1)=$ $\mathbb{E}_{U}\left(g_{i d}\right)$ is the expectation of the beta distribution with parameters $r$ and $n-r+1$. We thus have

$$
\operatorname{bel}(X=1)=\frac{r}{n+1} .
$$

Note that the above formula remains valid for $r=0$. Similarly, if $r<n, p l(X=1)=$ $\mathbb{E}_{V}\left(g_{i d}\right)$ is the expectation of the beta distribution with parameters $r+1$ and $n-r$. We thus have

$$
p l(X=1)=\frac{r+1}{n+1} .
$$

Again, this formula remains valid for $r=n$. The corresponding mass function $m_{X}$ regarding the value of $X$ is

$$
\begin{aligned}
m_{X}(\{0\}) & =\frac{n-r}{n+1} \\
m_{X}(\{1\}) & =\frac{r}{n+1} \\
m_{X}(\{0,1\}) & =\frac{1}{n+1} .
\end{aligned}
$$

\subsection{Least Commitment Principle}

The Least commitment Principle (LCP) [27], or Principle of Maximum Uncertainty [18] plays an important role in the theory of belief function, in a way similar to the principle of maximum entropy in Bayesian Probability Theory. As explained in [27], the LCP states that, given two belief functions compatible with a set of constraints, the most appropriate is the least informative.

To make this principle operational, it is necessary to define ways of comparing belief functions according to their information content. Several such partial orderings have been defined by Yager [35], Dubois and Prade [13], and Denœux [9]. We will focus here on the plausibility ordering $\sqsubseteq$ defined as follows for belief functions on $\mathbb{R}$ : we say that $m$ is more committed than $m^{\prime}$, and we write $m \sqsubseteq m^{\prime}$ if

$$
p l(A) \leq p l^{\prime}(A), \quad \forall A \in \mathcal{B}(\mathbb{R}) .
$$

As $\operatorname{bel}(A)=1-\operatorname{pl}(\bar{A})$ for all Borel set $A$, this is equivalent to the following condition:

$$
\operatorname{bel}(A) \geq \operatorname{bel}^{\prime}(A), \quad \forall A \in \mathcal{B}(\mathbb{R}) .
$$

It is clear that relation $\sqsubseteq$ is a partial ordering in the set of real mass functions.

Given two real numbers $a$ and $b$ such that $a<b$, let $\mathcal{M}([a, b])$ be the set of mass functions with support in $[a, b]$, i.e., such that all focal intervals are included in interval 
$[a, b]$. The greatest element of $\sqsubseteq$ in $\mathcal{M}([a, b])$ is the vacuous mass function on $[a, b]$, denoted as $m_{[a, b]}$ and defined by $m([a, b])=1$ and $m(I)=0$ for all intervals $I \neq[a, b]$. The corresponding plausibility function $p l_{[a, b]}$ verifies $p l_{[a, b]}(A)=1$ for all $A \in \mathcal{B}(\mathbb{R})$ such that $A \cap[a, b] \neq \emptyset$.

\section{Credal Orderings}

As recalled in Section 1, the definition of the stochasting ordering relation is based on the comparison of probabilities for each event $(x,+\infty)$, for any $x \in \mathbb{R}$. In the belief function framework, one associates to each such event no long a single numer, but an interval defined by its belief and plausibility values. One then has to replace the usual ordering relation between real numbers by an ordering relation between intervals. As several such orderings can be defined, we will obtain different extensions of stochastic ordering in the belief function framework.

\subsection{Definitions}

Let us consider two intervals $[a, b]$ and $\left[a^{\prime}, b^{\prime}\right]$. They can basically be in four positions $[16,12]$ :

1. $[a, b] \lesssim\left[a^{\prime}, b^{\prime}\right]$ if and only if $a \leq b^{\prime} ;$

2. $[a, b] \leqslant\left[a^{\prime}, b^{\prime}\right]$ if and only if $a \leq a^{\prime}$;

3. $[a, b] \gtrless\left[a^{\prime}, b^{\prime}\right]$ if and only if $b \leq b^{\prime}$;

4. $[a, b] \ll\left[a^{\prime}, b^{\prime}\right]$ if and only if $b \leq a^{\prime}$.

It is clear that $\lesssim$ is the weakest of these relations, while $\ll$ is the strongest one. The following implications hold:

$$
\begin{aligned}
& {[a, b] \ll\left[a^{\prime}, b^{\prime}\right] \Rightarrow[a, b] \leqslant\left[a^{\prime}, b^{\prime}\right] \Rightarrow[a, b] \lesssim\left[a^{\prime}, b^{\prime}\right],} \\
& {[a, b] \ll\left[a^{\prime}, b^{\prime}\right] \Rightarrow[a, b] \gtrless\left[a^{\prime}, b^{\prime}\right] \Rightarrow[a, b] \lesssim\left[a^{\prime}, b^{\prime}\right] .}
\end{aligned}
$$

To each of the above relations between intervals can be associated a relation between belief functions. More precisely, for any two mass functions $m$ and $m^{\prime}$ on $\mathbb{R}$, let us introduce the following definitions:

1. $m \lesssim m^{\prime}$ if and only if $\operatorname{bel}((x,+\infty)) \leq p l^{\prime}((x,+\infty)), \quad \forall x \in \mathbb{R}$;

2. $m \leqslant m^{\prime}$ if and only if $\operatorname{bel}((x,+\infty)) \leq \operatorname{bel}^{\prime}((x,+\infty)), \quad \forall x \in \mathbb{R}$;

3. $m<m^{\prime}$ if and only if $p l((x,+\infty)) \leq p l^{\prime}((x,+\infty)), \quad \forall x \in \mathbb{R}$;

4. $m \ll m^{\prime}$ if and only if $p l((x,+\infty)) \leq \operatorname{bel}^{\prime}((x,+\infty)), \quad \forall x \in \mathbb{R}$.

It is obvious that similar implications as in (34)-(35) hold for these four relations between mass functions. In the following, they will be referred to as the weak, lower, upper and strong credal orderings, respectively. Following the usual convention, the corresponding inverse relations will denoted as $\gtrsim, \geqslant,>$ and $\gg$, respectively. 


\subsection{Properties}

First of all, it is clear that the above four credal orderings boil down to stochastic ordering when $m$ and $m^{\prime}$ are Bayesian: they thus qualify as valid extensions of this concept in the belief function setting. The fact that credal orderings extend both stochastic ordering and interval orderings is consistent with the two complementary views of belief functions as generalized probability measures and generalized sets [13].

The credal oredrings introduced in this paper should not be confused with the informational orderings recalled in Section 2.3. However, the following proposition points to a relationship with the plausibility ordering $\sqsubseteq$.

Proposition 2 For any two mass function $m$ and $m^{\prime}$ on $\mathbb{R}$ :

$$
m \sqsubseteq m^{\prime} \Rightarrow\left\{\begin{array}{l}
m<m^{\prime} \\
m^{\prime} \leqslant m,
\end{array}\right.
$$

Proof. By definition, $m \sqsubseteq m^{\prime}$ means that $p l(A) \leq p l^{\prime}(A)$ for all Borel set $A$ or, equivalently, $\operatorname{bel}(A) \geq \operatorname{bel}^{\prime}(A)$ for all Borel set $A$. Consequently, $m \sqsubseteq m^{\prime}$ implies that $p l((x,+\infty)) \leq$ $p l^{\prime}((x,+\infty))$ and $\operatorname{bel}((x,+\infty)) \geq \operatorname{bel}^{\prime}((x,+\infty))$ for all $x \in \mathbb{R}$, i.e., $m<m^{\prime}$ and $m^{\prime} \leqslant m$.

The four basic credal orderings also admit interesting characterizations in terms of stochastic inequalities between probability measures associated to mass functions, as expressed in the following proposition.

Proposition 3 Let $m$ and $m^{\prime}$ be two mass functions, and let $[U, V]$ and $\left[U^{\prime}, V^{\prime}\right]$ be the corresponding random intervals. The following equivalences hold:

$$
\begin{aligned}
m \lesssim m^{\prime} & \Leftrightarrow U \leq_{\mathrm{st}} V^{\prime} \\
m \leqslant m^{\prime} & \Leftrightarrow U \leq_{\mathrm{st}} U^{\prime} \\
m \gtrless m^{\prime} & \Leftrightarrow V \leq_{\mathrm{st}} V^{\prime} \\
m \ll m^{\prime} & \Leftrightarrow V \leq_{\mathrm{st}} U^{\prime} .
\end{aligned}
$$

Proof. Direct from (6)-(7). For instance, we have bel $((x,+\infty))=1-p l((-\infty, x])=$ $1-F_{U}(x)$ and $b e l^{\prime}((x,+\infty))=1-p l^{\prime}((-\infty, x])=1-F_{U^{\prime}}(x)$ for all $x$. Hence, $m \leqslant m^{\prime}$ iff $F_{U} \geq F_{U^{\prime}}$, that is, iff $U \leq_{\text {st }} U^{\prime}$.

Lastly, the four credal orderings introduced above also admit a characterization in terms of lower and upper expectations. This is described in the following proposition.

Proposition 4 Let $m$ and $m^{\prime}$ be two mass functions, and let $\mathcal{G}$ denote the set of bounded, measurable and non decreasing real functions. Then we have:

$$
\begin{aligned}
m \lesssim m^{\prime} & \Leftrightarrow \quad \mathbb{E}_{m}(g) \leq \overline{\mathbb{E}}_{m^{\prime}}(g), \quad \forall g \in \mathcal{G} \\
m \leqslant m^{\prime} & \Leftrightarrow \quad \underline{\mathbb{E}}_{m}(g) \leq \underline{\mathbb{E}}_{m^{\prime}}(g), \quad \forall g \in \mathcal{G} \\
m \gtrless m^{\prime} & \Leftrightarrow \quad \overline{\mathbb{E}}_{m}(g) \leq \overline{\mathbb{E}}_{m^{\prime}}(g), \quad \forall g \in \mathcal{G} \\
m \ll m^{\prime} & \Leftrightarrow \quad \overline{\mathbb{E}}_{m}(g) \leq \underline{\mathbb{E}}_{m^{\prime}}(g), \quad \forall g \in \mathcal{G} .
\end{aligned}
$$

Proof. From Proposition 3, $m \leqslant m^{\prime} \Leftrightarrow U \leq_{\text {st }} U^{\prime}$. As recalled in Section 1, this is equivalent to $\mathbb{E}_{U}(g) \leq \mathbb{E}_{U^{\prime}}(g)$ for all $g \in \mathcal{G}$. Now, from Proposition $1, \mathbb{E}_{U}(g)=\underline{\mathbb{E}}_{m}(g)$ and $\mathbb{E}_{U^{\prime}}(g)=\underline{\mathbb{E}}_{m^{\prime}}(g)$ for all $g \in \mathcal{G}$, hence the result. The proof of the other equivalences is similar. 


\section{Least Committed Mass Function Subject to a Credal Or- dering Constraint}

Let $m$ be a real mass function with support in $[a, b]$ for some real numbers $a$ and $b$ such that $a<b$. Let $m^{\prime}$ be another mass function in $\mathcal{M}([a, b])$. Assume that $m$ is known, whereas we only know that $m^{\prime}$ is greater (or, alternatively, lower) than or equal to $m$, according to one of the credal ordering defined above. We would like to find the least committed mass function $m^{\prime}$ compatible with this constraint. Situations where problems of this kind may arise include the following:

- We consider two variables $X$ and $X^{\prime}$. Our beliefs on $X$ are represented by $m$. Additionally, we believe that $X^{\prime}$ tends to take greater values than $X$. How to quantify our beliefs on $X^{\prime}$ using a mass function?

- We consider one variable $X$ and two different contexts $C$ and $C^{\prime}$. When $C$ holds, our beliefs on $X$ are represented by $m$. When $C^{\prime}$ holds, we have less information and we are not able to express our belief on $X$ directly as a mass function. However, we believe that $X$ tends to take lower values than when $C$ holds. How to quantify our beliefs on $X$ in context $C^{\prime}$ ?

The above problem may be formalized as follows. Let

$$
\mathcal{S}_{R}^{[a, b]}(m)=\left\{m^{\prime} \in \mathcal{M}([a, b]) \mid m^{\prime} R m\right\}
$$

for some ordering relation $R$ in $\{\lesssim, \gtrsim, \leqslant, \geqslant, \gtrless,>, \ll, \gg\}$. We would like to find the leastcommitted element in $\mathcal{S}_{R}(m)$, if it exists. The solutions are described in the following subsections.

\subsection{Solution for $R \in\{\lesssim, \gtrsim,>, \leqslant\}$}

The cases where $R \in\{\lesssim, \gtrsim,>, \leqslant\}$ are relatively simple. We have seen in Section 2.3 that the vacuous mass function on $[a, b]$, denoted as $m_{[a, b]}$, is the greatest element in $\mathcal{M}([a, b])$ for the $\sqsubseteq$ relation, i.e, $m \sqsubseteq m_{[a, b]}$ for all $m \in \mathcal{M}([a, b])$. From Proposition 2 , it follows that the following relations hold for all $m \in \mathcal{M}([a, b])$ :

$$
m_{[a, b]} \leqslant m, \quad m_{[a, b]} \lesssim m, \quad m_{[a, b]}>m, \quad m_{[a, b]} \gtrsim m .
$$

We may conclude that $m_{[a, b]}$ is the least commited element in $\mathcal{S}_{R}^{[a, b]}(m)$ for $R \in\{\lesssim, \gtrsim,>$ $, \leqslant\}$ and all $m \in \mathcal{M}([a, b])$.

This trivial result shows that constraints of the form $m^{\prime} R m$ with $R \in\{\lesssim, \gtrsim,>, \leqslant\}$ for fixed $m$ are too weak to result in the selection of an informative, i.e., non vacuous mass function $m^{\prime}$. As will be shown, the four other types of credal inequality constraints lead to more useful solutions. These solutions are detailed in the following subsections, and summarized in Table 1.

\subsection{Constraint of the form $m^{\prime} \geqslant m$}

The following proposition shows that the problem of finding the least committed mass function $m^{\prime}$ in $\mathcal{M}([a, b])$ such that $m^{\prime} \geqslant m$ for given $m$ in $\mathcal{M}([a, b])$ has a simple solution. 
As this solution is consonant, it is more concisely described by its corresponding possibility distribution. This is done in Proposition 5. The expressions of the corresponding mass function in the discrete and continuous cases are then given in Propositions 7 and 8, respectively.

Proposition 5 The least committed element in $\mathcal{S}_{\geqslant}^{[a, b]}(m)$ exists and is unique. It is the consonant mass function $m \geqslant$ with possibility distribution $\pi \geqslant$ given by

$$
\pi_{\geqslant}(x)= \begin{cases}p l((-\infty, x]) & \text { if } x \leq b, \\ 0 & \text { if } x>b\end{cases}
$$

where $p l$ is the plausibility function associated to $m$.

Proof. Let $m^{\prime} \in \mathcal{M}([a, b])$. We have $m^{\prime} \in \mathcal{S}_{\geqslant}^{[a, b]}(m)$ iff it verifies the following constraints:

$$
\operatorname{bel}^{\prime}((x,+\infty)) \geq \operatorname{bel}((x,+\infty)), \quad \forall x \in \mathbb{R}
$$

or, equivalently,

$$
p l^{\prime}((-\infty, x]) \leq p l((-\infty ; x]), \quad \forall x \in \mathbb{R} .
$$

In particular, for $p l \geqslant$ defined by $(44)$, we have

$$
p l_{\geqslant}((-\infty, x])=\sup _{y \leq x} \pi_{\geqslant}(y)=p l((-\infty, x]) .
$$

Consequently, condition (45) is verified by $m \geqslant$. To show that $m \geqslant$ is least committed, we need to prove that if $m^{\prime} \in \mathcal{S}_{\geqslant}^{[a, b]}(m)$, then $m^{\prime}$ is more committed than $m_{\geqslant}$, i.e., $p l^{\prime}(A) \leq$ $p l \geqslant(A)$ for all $A$. So, let $m^{\prime}$ be an arbitrary mass function in $\mathcal{S}_{\geqslant}^{[a, b]}(m)$. Let $A$ be a Borel set on $\mathbb{R}$. If $A \cap[a, b]=\emptyset$, then $p l^{\prime}(A)=p l \geqslant(A)=0$, since $m^{\prime} \in \mathcal{M}([a, b])$. Assume that $A \cap[a, b] \neq \emptyset$, and let $s=\sup (A \cap[a, b])$. We have $A \cap[a, b] \subseteq(-\infty, s]$ and, consequently

$$
p l^{\prime}(A)=p l^{\prime}(A \cap[a, b]) \leq p l^{\prime}((-\infty, s]) \leq p l((-\infty ; s])=\pi_{\geqslant}(s) .
$$

Since function $\pi_{\geqslant}$is non decreasing and right-continuous on $(-\infty, b]$, (where it is identical to the cdf of $U)$, we have $\pi_{\geqslant}(s)=\sup _{x \leq s} \pi_{\geqslant}(x)=p l_{\geqslant}(A)$. Consequently,

$$
p l^{\prime}(A) \leq p l \geqslant(A), \quad \forall A \in \mathcal{B}(\mathbb{R}),
$$

which shows that $m \geqslant$ is indeed the least committed mass function in $\mathcal{S}_{\geqslant}^{[a, b]}(m)$.

The following propositions give the expressions of the random interval $\left[U_{\geqslant}, V_{\geqslant}\right]$as well as the mass function $m \geqslant$ associated to $\pi \geqslant$, in the discrete and continuous cases.

Proposition 6 Let $\left[U_{\geqslant}, V_{\geqslant}\right]$be the random interval associated to $\pi_{\geqslant}$defined in Proposition 5. The cdfs of $U \geqslant$ and $V \geqslant$ are

$$
\begin{aligned}
& F_{U \geqslant}(x)=F_{U}(x), \\
& F_{V_{\geqslant}}(x)=H(x-b),
\end{aligned}
$$

for all $x \in \mathbb{R}$, where $H$ is the Heaviside step function defined by $H(u)=1$ if $u \geq 0$ and $H(u)=0$ otherwise. 
Proof. By constrution, $p l_{\geqslant}((-\infty, x])=p l((-\infty, x])$ for all $x$, hence $F_{U \geqslant}=F_{U}$. Now,

$$
\begin{aligned}
F_{V_{\geqslant}}(x) & =b e l_{\geqslant}((-\infty, x]) \\
& =1-p l_{\geqslant}((x,+\infty)) \\
& =1-\sup _{y>x} \pi_{\geqslant}(y) \\
& = \begin{cases}0 & \text { if } x<b \\
1 & \text { if } x \geq b .\end{cases}
\end{aligned}
$$

Proposition 7 Let $m$ be a discrete mass function with focal intervals $\left[u_{i}, v_{i}\right] \subseteq[a, b]$, $i=1, \ldots, n$ and masses $m\left(\left[u_{i}, v_{i}\right]\right)=m_{i}, i=1, \ldots, n$. Let $u_{(1)}<u_{(2)}<\ldots<u_{(\ell)}$ be the $\ell$ distinct values of $u_{1}, \ldots, u_{n}$. Then $m \geqslant$ defined in Proposition 5 is a discrete mass function with the following expression:

$$
m \geqslant\left(\left[u_{(j)}, b\right]\right)=\sum_{\left\{i \mid u_{i}=u_{(j)}\right\}} m_{i}, \quad j=1, \ldots, \ell
$$

Proof. The mass function $m \geqslant$ defined by (52) is consonant. Let $\pi \geqslant$ be the corresponding possibility distribution. If $x>b$ then $\pi \geqslant(x)=0$. If $x \leq b$,

$$
\begin{aligned}
\pi_{\geqslant}(x) & =\sum_{\left\{j \mid u_{(j)} \leq x\right\}} m_{\geqslant}\left(\left[u_{(j)}, b\right]\right) \\
& =\sum_{\left\{j \mid u_{(j)} \leq x\right\}} \sum_{\left\{i \mid u_{i}=u_{(j)}\right\}} m_{i} \\
& =\sum_{\left\{i \mid u_{i} \leq x\right\}} m_{i}=p l((-\infty, x]),
\end{aligned}
$$

which is identical to (44).

Proposition 8 Let $m$ be a continuous mass function. Then $m \geqslant$ defined in Proposition 5 is a continuous mass function with the following expression:

$$
m_{\geqslant}([u, b])=f_{U}(u), \quad \forall u \leq b .
$$

Proof. Let $m_{\geqslant}$be defined by (53). It is consonant. Let $\pi_{\geqslant}$be the corresponding possibility distribution. If $x>b$ then $\pi_{\geqslant}(x)=0$. If $x \leq b$,

$$
\begin{aligned}
\pi_{\geqslant}(x) & =\int_{a}^{x} m_{\geqslant}([u, b]) \mathrm{d} u \\
& =\int_{a}^{x} f_{U}(u) \mathrm{d} u \\
& =F_{U}(x) .
\end{aligned}
$$

Then, by Equation (7), we have

$$
F_{U}(x)=p l((-\infty, x])
$$


and hence

$$
\pi_{\geqslant}(x)=p l((-\infty, x])
$$

which is identical to (44).

Example 3 Let us come back to Example 1. Assume that $m$ represents the available information regarding the failure probability $p$ of a component in standard operating condition, and we want to assess our beliefs regarding the failure probability $p^{\prime}$ of the same component in a different, more stringent environment, for which we have no data. We only know that the failure probability in this new environment tends to be higher than the failure probability in standard operating condition. If this piece of knowledge is modelled using the lower ordering and if we accept the least commitment principle, then our knowledge regarding $p^{\prime}$ should be represented by the mass function $m \geqslant$ derived from $m$.

Let us first assume that $m$ is a discrete mass function constructed from expert opinion using the method of consonant intervals. Furthermore, let us assume that the following numerical values were obtained:

$$
\begin{gathered}
m([0.1,0.15])=0.3, \quad m([0.05,0.3])=0.4, \\
m([0,0.3])=0.2, \quad m([0,1])=0.1 .
\end{gathered}
$$

Using (52), we get

$$
\begin{gathered}
m_{\geqslant}([0.1,1])=0.3, \quad m_{\geqslant}([0.05,1])=0.4, \\
m_{\geqslant}([0,1])=0.2+0.1=0.3 .
\end{gathered}
$$

If $\mathcal{F} \geqslant$ denotes the set of focal sets of $m_{\geqslant}$, then, by definition of a possibility distribution, we have

$$
\pi_{\geqslant}(p)=\sum_{I \in \mathcal{F} \geqslant, p \in I} m_{\geqslant}(I)
$$

and we easily obtain

$$
\pi_{\geqslant}(p)= \begin{cases}0 & \text { if } p<0 \text { or } p>1 \\ 0.3 & \text { if } 0 \leq p<0.05 \\ 0.7 & \text { if } 0.05 \leq p<0.1 \\ 1 & \text { if } 0.1 \leq p \leq 1\end{cases}
$$

Let us now assume that $m$ is the continuous mass function resulting from the observation of $r$ failures out of $n$ trials and defined by (12)-(14). If $r=0$, then $f_{U}$ is a Dirac delta function centered at zero, and we get $m_{\geqslant}([0,1])=1$. If $r>0, f_{U}$ is given by (15). Using (53), we get

$$
m_{\geqslant}([u, 1])=\frac{n !}{(r-1) !(n-r) !} u^{r-1}(1-u)^{n-r}, \quad \forall u \in[0,1] .
$$

As in Example 2, we can also compute the belief and plausibility that the component will fail in the new environment. Let $S E$ denote the proposition that the component will be used in the new, more stringent environment. We have

$$
\begin{aligned}
\operatorname{bel}(X=1 \mid S E) & =\underline{\mathbb{E}}_{m \geqslant}\left(g_{i d}\right)=\frac{r}{n+1} \\
p l(X=1 \mid S E) & =\overline{\mathbb{E}}_{m \geqslant}\left(g_{i d}\right)=1 .
\end{aligned}
$$


The corresponding mass function is

$$
\begin{aligned}
m_{X}(\{0\} \mid S E) & =0 \\
m_{X}(\{1\} \mid S E) & =\frac{r}{n+1} \\
m_{X}(\{0,1\} \mid S E) & =\frac{n-r+1}{n+1},
\end{aligned}
$$

which can be compared to (29)-(31).

\subsection{Constraint of the form $m^{\prime}<m$}

The problem of finding the least committed mass function $m^{\prime}$ subject to a constraint of the form $m^{\prime}<m$ for fixed $m$ is symmetrical to that described in the previous subsection. We first give the expression of the possibility distribution in Proposition 9, and then provide the expressions for the corresponding mass functions in the discrete and continuous cases in Propositions 11 and 12, respectively.

Proposition 9 The least committed element in $\mathcal{S}_{\gtrless}^{[a, b]}(m)$ exists and is unique. It is the consonant mass function $m_{\gtrless}$ with possibility distribution $\pi_{\gtrless}$ given by

$$
\pi_{\gtrless}(x)= \begin{cases}0 & \text { if } x<a, \\ p l([x,+\infty)) & \text { if } x \geq a,\end{cases}
$$

where $p l$ is the plausibility function associated to $m$.

Proof. Let $m^{\prime} \in \mathcal{M}([a, b])$. We have $m^{\prime} \in \mathcal{S}_{\gtrless}^{[a, b]}(m)$ iff it verifies the following constraints:

$$
p l^{\prime}((x,+\infty)) \leq p l((x,+\infty)), \quad \forall x \in \mathbb{R} .
$$

Let us show that the above condition is verified for $m_{\gtrless}$ defined by (57). We have

$$
p l_{\gtrless}((x,+\infty))=\sup _{y>x} \pi_{\gtrless}(y)=\sup _{y>x} p l([y,+\infty))=p l((x,+\infty)),
$$

for all real $x$. Furthermore,

$$
p l_{\gtrless}([x,+\infty))=\sup _{y \geq x} \pi_{\gtrless}(y)=\pi_{\gtrless}(x)=p l([x,+\infty)),
$$

for all real $x$. To show that $m_{\gtrless}$ is least committed, we need to prove that if $m^{\prime} \in \mathcal{S}_{\gtrless}^{[a, b]}(m)$, then $m^{\prime}$ is more committed than $m_{₹}$, i.e., $p l^{\prime}(A) \leq p l_{₹}(A)$ for all $A$. So, let $m^{\prime}$ be an arbitrary mass function in $\mathcal{S}_{\gtrless}^{[a, b]}(m)$. Let $A$ be a Borel set on $\mathbb{R}$. If $A \cap[a, b]=\emptyset$, then $p l^{\prime}(A)=0$, since $m^{\prime} \in \mathcal{M}([a, b])$. Assume that $A \cap[a, b] \neq \emptyset$, and let $i=\inf A \cap[a, b]$. We have $A \cap[a, b] \subseteq[i,+\infty)$ and, consequently

$$
p l^{\prime}(A)=p l^{\prime}(A \cap[a, b]) \leq p l^{\prime}([i,+\infty)) \leq p l([i,+\infty))=\pi_{\gtrless}(i) .
$$

Since function $\pi_{\gtrless}$ is non increasing and left-continuous on $[a,+\infty)$, we have $\pi_{\gtrless}(i)=$ $\sup _{x \geq i} \pi_{\gtrless}(x)=p l_{\gtrless}(A)$. Consequently,

$$
p l^{\prime}(A) \leq p l_{\gtrless}(A), \quad \forall A \in \mathcal{B}(\mathbb{R}),
$$

which shows that $m_{\gtrless}$ is indeed the least committed mass function in $\mathcal{S}_{\gtrless}^{[a, b]}(m)$.

The following three propositions are the direct counterparts of Propositions 6, 7 and 8. The proofs are immediate. 
Proposition 10 Let $\left[U_{₹}, V_{₹}\right]$ be the random interval associated to $\pi_{\gtrless}$ defined in Proposition 9. The cdfs of $U_{\gtrless}$ and $V_{\gtrless}$ are

$$
\begin{aligned}
& F_{U_{乏}}(x)=H(x-a), \\
& F_{V_{乏}}(x)=F_{V}(x),
\end{aligned}
$$

for all $x \in \mathbb{R}$.

Proposition 11 Let $m$ be a discrete mass function with focal intervals $\left[u_{i}, v_{i}\right] \subseteq[a, b]$, $i=1, \ldots, n$ and masses $m\left(\left[u_{i}, v_{i}\right]\right)=m_{i}, i=1, \ldots, n$. Let $v_{(1)}<v_{(2)}<\ldots<v_{(q)}$ be the $q$ distinct values of $v_{1}, \ldots, v_{n}$. Then $m_{\gtrless}$ defined in Proposition 9 is a discrete mass function with the following expression:

$$
m_{\gtrless}\left(\left[a, v_{(j)}\right]\right)=\sum_{\left\{i \mid v_{i}=v_{(j)}\right\}} m_{i}, \quad j=1, \ldots, q .
$$

Proposition 12 Let $m$ be a continuous mass function. Then $m_{\gtrless}$ defined in Proposition 9 is a continuous mass function with the following expression:

$$
m_{\gtrless}([a, v])=f_{V}(v), \quad \forall v \geq a .
$$

Example 4 Let us continue Example 3, assuming now that the piece of equipement will be used in a more favorable environment in which the probability of failure $p^{\prime}$ is expected to be lower than the one corresponding to standard operating conditions. In the discrete case, we get, with the same figures as in Example 3:

$$
\begin{gathered}
m_{\gtrless}([0,0.15])=0.3, \quad m_{\gtrless}([0,0.3])=0.4+0.2=0.6, \\
m_{\gtrless}([0,1])=0.1 .
\end{gathered}
$$

The corresponding possibility distribution is given by (57):

$$
\pi_{\gtrless}(p)= \begin{cases}0 & \text { if } p<0 \text { or } p>1 \\ 1 & \text { if } 0 \leq p \leq 0.15 \\ 0.7 & \text { if } 0.15<p \leq 0.3 \\ 0.1 & \text { if } 0.3<p \leq 1\end{cases}
$$

Let us now consider the continuous case where $m$ is given by (12)-(14). If $r=n$, then $f_{V}$ is the Dirac delta function centered at one. If $r<n$ then $f_{V}$ is given by (16) and we get, using (61):

$$
m_{\gtrless}([0, v])=\frac{n !}{r !(n-r-1) !} v^{r}(1-v)^{n-r-1}, \quad \forall v \geq 0 .
$$

Let $F E$ denote the proposition that the piece of equipment will be used in a more favorable environment than the standard operating conditions. We have

$$
\begin{aligned}
\operatorname{bel}(X=1 \mid F E) & =\underline{\mathbb{E}}_{m_{\gtrless}}\left(g_{i d}\right)=0 \\
p l(X=1 \mid F E) & =\overline{\mathbb{E}}_{m_{\gtrless}}\left(g_{i d}\right)=\frac{r+1}{n+1} .
\end{aligned}
$$


The corresponding mass function is

$$
\begin{aligned}
m_{X}(\{0\} \mid F E) & =\frac{n-r}{n+1} \\
m_{X}(\{1\} \mid F E) & =0 \\
m_{X}(\{0,1\} \mid F E) & =\frac{r+1}{n+1},
\end{aligned}
$$

which, again, can be compared to (29)-(31).

\subsection{Constraint of the form $m^{\prime} \gg m$}

In this subsection, we give the counterpart of the result presented in Section 4.2, for a constraint of the form $m^{\prime} \gg m$ for given $m$. As we shall see, the solution is quite similar to that presented in Section 4.2. The proofs are similar and will only be sketched.

Proposition 13 The least committed element in $\mathcal{S}_{\gg}^{[a, b]}(m)$ exists and is unique. It is the consonant mass function $m_{\gg}$ with possibility distribution $\pi_{\gg}$ given by

$$
\pi_{\gg}(x)= \begin{cases}\operatorname{bel}((-\infty, x]) & \text { if } x \leq b, \\ 0 & \text { if } x>b,\end{cases}
$$

where bel is the belief function associated to $m$.

Proof. Let $m^{\prime} \in \mathcal{M}([a, b])$. We have $m^{\prime} \in \mathcal{S}_{\gg}^{[a, b]}(m)$ iff it verifies the following constraints:

$$
p l((x,+\infty)) \leq b e l^{\prime}((x,+\infty)), \quad \forall x \in \mathbb{R}
$$

or, equivalently,

$$
p l^{\prime}((-\infty, x]) \leq \operatorname{bel}((-\infty ; x]), \quad \forall x \in \mathbb{R} .
$$

In particular, this condition is verified by $m_{\gg}$ defined by (65). For an arbitrary $m^{\prime} \in$ $\mathcal{S}_{\gg}^{[a, b]}(m)$, the largest value that can be given to $p l^{\prime}((-\infty, x])$ is thus bel $((-\infty, x])$. Assume that $p l^{\prime}((-\infty, x])=\operatorname{bel}((-\infty ; x])$, for all $x \in \mathbb{R}$. Using the same line of reasoning as for the proof of Proposition 5, it can be shown that $m_{\gg}$ is the least committed mass function verifying these constraints.

The following propositions give the expressions of the random interval $\left[U_{\gg}, V_{\gg}\right]$ as well as the mass function $m_{\gg}$, in the discrete and continuous cases.

Proposition 14 Let $\left[U_{\gg}, V_{\gg}\right]$ be the random interval associated to $\pi_{\gg}$ defined in Proposition 13. The cdfs of $U_{\gg}$ and $V_{\gg}$ are

$$
\begin{aligned}
& F_{U_{\gg}}(x)=F_{V}(x), \\
& F_{V_{\gg}}(x)=H(x-b),
\end{aligned}
$$

for all $x \in \mathbb{R}$.

Proof. The proof is identical to that of Proposition 6. 
Proposition 15 Let $m$ be a discrete mass function with focal intervals $\left[u_{i}, v_{i}\right] \subseteq[a, b]$, $i=1, \ldots, n$ and masses $m\left(\left[u_{i}, v_{i}\right]\right)=m_{i}, i=1, \ldots, n$. Let $v_{(1)}<v_{(2)}<\ldots<v_{(q)}$ be the $q$ distinct values of $v_{1}, \ldots, v_{n}$. Then $m_{\gg}$ defined in Proposition 13 is a discrete mass function with the following expression:

$$
m_{\gg}\left(\left[v_{(j)}, b\right]\right)=\sum_{\left\{i \mid v_{i}=v_{(j)}\right\}} m_{i}, \quad j=1, \ldots, q
$$

Proof. It can be checked that the mass function $m_{\gg}$ defined by (68) is consonant, and that the corresponding possibility distribution $\pi_{\gg}$ is identical to (65).

Proposition 16 Let $m$ be a continuous mass function. Then $m_{\gg}$ defined in Proposition 13 is a continuous mass function with the following expression:

$$
m_{\gg}([u, b])=f_{V}(u), \quad \forall u \leq b .
$$

Proof. Let $m_{\gg}$ be the mass function defined by (69). It is consonant. Let $\pi_{\gg}$ be the corresponding possibility distribution. If $x>b$ then $\pi_{\gg}(x)=0$. If $x \leq b$,

$$
\begin{aligned}
\pi_{\gg}(x) & =\int_{a}^{x} m_{\gg}([u, b]) \mathrm{d} u \\
& =\int_{a}^{x} f_{V}(u) \mathrm{d} u \\
& =F_{V}(x) .
\end{aligned}
$$

Then, by Equation (6), we have

$$
F_{V}(x)=\operatorname{bel}((-\infty, x])
$$

and hence

$$
\pi_{\gg}(x)=\operatorname{bel}((-\infty, x])
$$

which is identical to (65).

Example 5 Let us again return to our reliability analysis problem. As in Example 3, $m$ is a mass function on the failure probability $p$ of a component in standard operating condition, and we want to assess our beliefs regarding the failure probability $p^{\prime}$ of the same component in a more stringent environment. As before, we only know that the failure probability in this new environment tends to be higher than the the failure probability in standard operating condition. Assume that this piece of knowledge is now modelled using the strong ordering.

Let us first consider the case where $m$ is discrete, with the same numerical values as in Example 1. Using (68), we get

$$
\begin{gathered}
m_{\gg}([0.15,1])=0.3, \quad m_{\gg}([0.3,1])=0.4+0.2=0.6, \\
m_{\gg}(\{1\})=0.1 .
\end{gathered}
$$


The corresponding possibility distribution is given by (65):

$$
\pi_{\gg}(p)= \begin{cases}0 & \text { if } p<0.15 \text { or } p>1 \\ 0.3 & \text { if } 0.15 \leq p<0.3 \\ 0.9 & \text { if } 0.3 \leq p<1 \\ 1 & \text { if } p=1\end{cases}
$$

Let us now assume that $m$ is continuous and defined by (12)-(14). If $r=n$, then $f_{V}$ is a Dirac delta function centered at one, and we get $m_{\gg}(\{1\})=1$. If $r<n, f_{V}$ is given by (16). Using (69), we get

$$
m_{\gg}([v, 1])=\frac{n !}{r !(n-r-1) !} v^{r}(1-v)^{n-r-1}, \quad \forall v \in[0,1] .
$$

As before, let $S E$ denote the proposition that the component will be used in the more stringent environment. We have

$$
\begin{aligned}
\operatorname{bel}(X=1 \mid S E) & =\underline{\mathbb{E}}_{m \gg}\left(g_{i d}\right)=\frac{r+1}{n+1} \\
p l(X=1 \mid S E) & =\overline{\mathbb{E}}_{m_{\gg}}\left(g_{i d}\right)=1 .
\end{aligned}
$$

The corresponding mass function is

$$
\begin{aligned}
m_{X}(\{0\} \mid S E) & =0 \\
m_{X}(\{1\} \mid S E) & =\frac{r+1}{n+1} \\
m_{X}(\{0,1\} \mid S E) & =\frac{n-r}{n+1}
\end{aligned}
$$

which can be compared to (29)-(31) and (54)-(56). In particular, we observe that, if the piece of equipment has failed in all trials under the standard operating conditions $(r=n)$, we find that $m_{X}(\{1\} \mid S E)=1$, i.e., we are sure that the same piece of equipment will fail in the more stringent environment. This result is somewhat paradoxical, which suggests that the constraint imposed by the use of the strong credal ordering is too stringent in this example.

\subsection{Constraint of the form $m^{\prime} \ll m$}

The solution for the case where $R$ is equal to $\ll$ is symmetrical to that presented in the previous section, and somewhat similar to that for $₹$ presented in Section 4.3. The results will be stated here without proof, for completeness.

Proposition 17 The least committed element in $\mathcal{S}_{\ll}^{[a, b]}(m)$ exists and is unique. It is the consonant mass function $m_{\ll}$ with possibility distribution $\pi_{\ll}$ given by

$$
\pi_{\ll}(x)= \begin{cases}0 & \text { if } x<a, \\ \operatorname{bel}([x,+\infty))) & \text { if } x \geq a,\end{cases}
$$

where bel is the belief function associated to $m$. 
Proof. The proof is similar to those of Propositions 5 and 13.

The following propositions are the counterparts of Propositions 14, 15 and 16. Their proofs are quite similar.

Proposition 18 Let $\left[U_{\ll}, V_{\ll}\right]$ be the random interval associated to $\pi_{\ll}$ defined in Proposition 17. The cdfs of $U_{\ll}$ and $V_{\ll}$ are

$$
\begin{aligned}
& F_{U_{\ll}}(x)=H(x-a), \\
& F_{V_{\ll}}(x)=F_{U}(x),
\end{aligned}
$$

for all $x \in \mathbb{R}$.

Proposition 19 Let $m$ be a discrete mass function with focal intervals $\left[u_{i}, v_{i}\right] \subseteq[a, b]$, $i=1, \ldots, n$ and masses $m\left(\left[u_{i}, v_{i}\right]\right)=m_{i}, i=1, \ldots, n$. Let $u_{(1)}<u_{(2)}<\ldots<u_{(\ell)}$ be the $\ell$ distinct values of $u_{1}, \ldots, u_{n}$. Then $m_{\ll}$ defined in Proposition 17 is a discrete mass function with the following expression:

$$
m_{\ll}\left(\left[a, u_{(j)}\right]\right)=\sum_{\left\{i \mid u_{i}=u_{(j)}\right\}} m_{i}, \quad j=1, \ldots, \ell
$$

Proposition 20 Let $m$ be a continuous mass function. Then $m_{\ll}$ defined in Proposition 17 is a continuous mass function with the following expression:

$$
m_{\ll}([a, v])=f_{U}(v), \quad \forall v \geq a .
$$

Example 6 To complete our study, let us continue the same problem as in Example 4, in which it was assumed that the piece of equipement will be used in a more favorable environment. The probability of failure $p^{\prime}$ is then expected to be lower than the one corresponding to standard operating conditions. Here, this piece of knowledge will be represented using the strong credal ordering. In the discrete case, we get, with the same figures as in Example 3:

$$
\begin{gathered}
m_{\ll}([0,0.1])=0.3, \quad m_{\ll}([0,0.05])=0.4, \\
m_{\ll}(\{0\})=0.2+0.1=0.3 .
\end{gathered}
$$

The corresponding possibility distribution is given by (70):

$$
\pi_{\ll}(p)= \begin{cases}0 & \text { if } p<0 \text { or } p>0.1 \\ 1 & \text { if } p=0 \\ 0.7 & \text { if } 0<p \leq 0.05 \\ 0.3 & \text { if } 0.05<p \leq 0.1\end{cases}
$$

Let us now consider the continuous case where $m$ is given by (12)-(14). If $r=0$, then $f_{U}$ is the Dirac delta function centered at zero, and we have $m_{\ll}(\{0\})=1$. If $r>0$ then $f_{U}$ is given by (15) and we get, using (74):

$$
m_{\ll}([0, u])=\frac{n !}{(r-1) !(n-r) !} u^{r-1}(1-u)^{n-r}, \quad \forall u \in[0,1] .
$$


Denoting, as before, by $F E$ the proposition that the piece of equipment will be used in a more favorable environment than the standard operating conditions, we have

$$
\begin{aligned}
\operatorname{bel}(X=1 \mid F E) & =\underline{\mathbb{E}}_{m_{\ll}}\left(g_{i d}\right)=0 \\
p l(X=1 \mid F E) & =\overline{\mathbb{E}}_{m_{\ll}}\left(g_{i d}\right)=\frac{r}{n+1} .
\end{aligned}
$$

The corresponding mass function is

$$
\begin{aligned}
m_{X}(\{0\} \mid F E) & =\frac{n-r+1}{n+1} \\
m_{X}(\{1\} \mid F E) & =0 \\
m_{X}(\{0,1\} \mid F E) & =\frac{r}{n+1},
\end{aligned}
$$

which can be compared to (29)-(31) and (62)-(64). As in Example 6, we may remark that the obtained mass function seems too specific: if $r=0$, i.e., the piece of equipement never failed in standard operating conditions out of $n$ trials, then we find $m_{X}(\{0\} \mid F E)=1$, corresponding to the certainty that the piece of equipement will not fail in future use under less stringent conditions. This again suggests that the use of the strong credal ordering is not fully appropriate in that case.

\section{Conclusions}

The usual concept of stochastic ordering between probability distributions has been extended to belief functions on the real line, resulting in four distinct ordering relations corresponding to the four basic orderings between intervals. Each of these orderings admits a characterization in terms of lower or upper expectations, generalizing a corresponding result in the case of stochastic ordering and standard expectation. Note that the approach described here is quite different from that presented in [25], where Dempster-Shafer theory is used to compare real intervals or fuzzy numbers, and the result of the comparison is given in the form of a belief interval or a fuzzy number.

The problem of finding the least committed belief function subject to a credal ordering constraint has then been studied in detail for each of the four basic credal orderings. In each case, the solution has been found to be a consonant belief function that can be described by a possibility distribution. The obtained formula may be used to model uncertain ordinal information such as " $X$ is likely to be greater than $Y$ " or " $X$ tends to take smaller values in context $C_{1}$ than in context $C_{2}$ ", in the belief function framework. More complex constraints such as " $X$ is likely to be greater than $Y$ and smaller than $Z$ " could also be considered, using a similar approach.

The initial motivation of this work arose from a novelty detection application $[2,3]$, in which we have data for only one state $S$ of a system under study, and we want to design a decision rule that detects the occurence of unknown states. Typically, one designs a "novelty measure" $X$ whose probability distribution given $S$ can be learnt from data, and which is expected to take higher values when the system is in any of the unknown states. Using the concepts introduced in this paper, it is possible to model our knowledge of $X$ when the system is in an unknown state as a belief function, and to derive a decision rule using the Generalized Bayes Theorem [27]. More results in this direction will be reported in future publications. 


\section{References}

[1] R. G. Almond. Graphical belief models. Chapman and Hall, London, 1995.

[2] A. Aregui and T. Denœux. Novelty detection in the belief functions framework. In Proceedings of IPMU '06, volume 1, pages 412-419, Paris, July 2006.

[3] A. Aregui and T. Denœux. Fusion of one-class classifiers in the belief function framework. In Proceedings of the 10th Int. Conf. on Information Fusion, Quebec, Canada, July 2007.

[4] E. Côme, L. Oukhellou, T. Denœux, and P. Aknin. Learning from partially supervised data using mixture models and belief functions. Pattern Recognition, 42(3):334-348, 2009 .

[5] A. P. Dempster. New methods for reasoning towards posterior distributions based on sample data. Annals of Mathematical Statistics, 37:355-374, 1966.

[6] A. P. Dempster. Upper and lower probabilities induced by a multivalued mapping. Annals of Mathematical Statistics, 38:325-339, 1967.

[7] A. P. Dempster. Upper and lower probabilities generated by a random closed interval. Annals of Mathematical Statistics, 39(3):957-966, 1968.

[8] A. P. Dempster. The Dempster-Shafer calculus for statisticians. International Journal of Approximate Reasoning, 48(2):365-377, 2008.

[9] T. Denœux. Conjunctive and disjunctive combination of belief functions induced by non distinct bodies of evidence. Artificial Intelligence, 172:234-264, 2008.

[10] T. Denœux and M.-H. Masson. EVCLUS: Evidential clustering of proximity data. IEEE Trans. on Systems, Man and Cybernetics B, 34(1):95-109, 2004.

[11] T. Denœux and P. Smets. Classification using belief functions: the relationship between the case-based and model-based approaches. IEEE Transactions on Systems, Man and Cybernetics B, 36(6):1395-1406, 2006.

[12] D. Dubois, E. Kerre, R. Mesiar, and H. Prade. Fuzzy interval analysis. In D. Dubois and H. Prade, editors, Fundamentals of Fuzzy sets, pages 483-581. Kluwer Academic Publishers, Boston, 2000.

[13] D. Dubois and H. Prade. A set-theoretic view of belief functions: logical operations and approximations by fuzzy sets. International Journal of General Systems, 12(3):193-226, 1986.

[14] D. Dubois and H. Prade. Possibility Theory: An approach to computerized processing of uncertainty. Plenum Press, New-York, 1988.

[15] S. Ferson, V. Kreinovitch, L. Ginzburg, D. S. Myers, and K. Sentz. Constructing probability boxes and Dempster-Shafer structures. Technical Report SAND20024015, Sandia National Laboratories, Albuquerque, NM, 2003. 
[16] P. C. Fishburn. Interval orders and interval graphs. Wiley, New-York, 1985.

[17] M. Ha-Duong. Hierarchical fusion of expert opinions in the Transferable Belief Model. International Journal of Approximate Reasoning, 49(3):555-574, 2008.

[18] G. J. Klir and M. J. Wierman. Uncertainty-Based Information. Elements of Generalized Information Theory. Springer-Verlag, New-York, 1999.

[19] J. Kohlas and P.-A. Monney. An algebraic theory for statistical information based on the theory of hints. International Journal of Approximate Reasoning, 48(2):Pages 378-398, 2008.

[20] S. Kotz and N. L. Johnson. Discrete Distributions. Houghton Mifflin Company, Boston, 1969.

[21] E. L. Lehman. Ordered families of distributions. The Annals of Mathematical Statistics, 26(3):399-419, 1955.

[22] M.-H. Masson and T. Denœux. ECM: an evidential version of the fuzzy c-means algorithm. Pattern Recognition, 41(4):1384-1397, 2008.

[23] S. Petit-Renaud and T. Denœux. Nonparametric regression analysis of uncertain and imprecise data using belief functions. International Journal of Approximate Reasoning, 35(1):1-28, 2004.

[24] B. Quost, T. Denœux, and M.-H. Masson. Pairwise classifier combination using belief functions. Pattern Recognition Letters, 28(5):644-653, 2007.

[25] P. Sevastianov. Numerical methods for interval and fuzzy number comparison based on the probabilistic approach and Dempster-Shafer theory. Information Sciences, 177(21):4645-4661, 2007.

[26] G. Shafer. A mathematical theory of evidence. Princeton University Press, Princeton, N.J., 1976.

[27] P. Smets. Belief functions: the disjunctive rule of combination and the generalized Bayesian theorem. International Journal of Approximate Reasoning, 9:1-35, 1993.

[28] P. Smets. The Transferable Belief Model for quantified belief representation. In D. M. Gabbay and P. Smets, editors, Handbook of Defeasible reasoning and uncertainty management systems, volume 1, pages 267-301. Kluwer Academic Publishers, Dordrecht, 1998.

[29] P. Smets. Belief functions on real numbers. International Journal of Approximate Reasoning, 40(3):181-223, 2005.

[30] P. Smets and R. Kennes. The Transferable Belief Model. Artificial Intelligence, 66:191-243, 1994.

[31] Y.-M. Wang, J.-B. Yang, D.-L. Xu, and K.-S. Chin. On the combination and normalization of interval-valued belief structures. Information Sciences, 177(5):1230-1247, 2007. 
[32] L. A. Wasserman. Belief functions and statistical evidence. The Canadian Journal of Statistics, 18(3):183-196, 1990.

[33] W. Whitt. Stochastic ordering. In S. Kotz and N. L. Johnson, editors, Encyclopedia of the Statistical Sciences, volume 8, pages 832-836. Wiley, New York, 1988.

[34] W.-Z. Wu. Attribute reduction based on evidence theory in incomplete decision systems. Information Sciences, 178(5):1355-1371, 2008.

[35] R. R. Yager. The entailment principle for Dempster-Shafer granules. Int. J. of Intelligent Systems, 1:247-262, 1986.

[36] R. R. Yager. Cumulative distribution functions from Dempster-Shafer belief structures. IEEE Trans. on Systems, Man and Cybernetics B, 34(5):2080-2087, 2004.

[37] R. R. Yager and L. Liu, editors. Classic Works of the Dempster-Shafer Theory of Belief Functions. Springer, Heidelberg, 2008.

[38] L. A. Zadeh. Fuzzy sets as a basis for a theory of possibility. Fuzzy Sets and Systems, 1:3-28, 1978. 


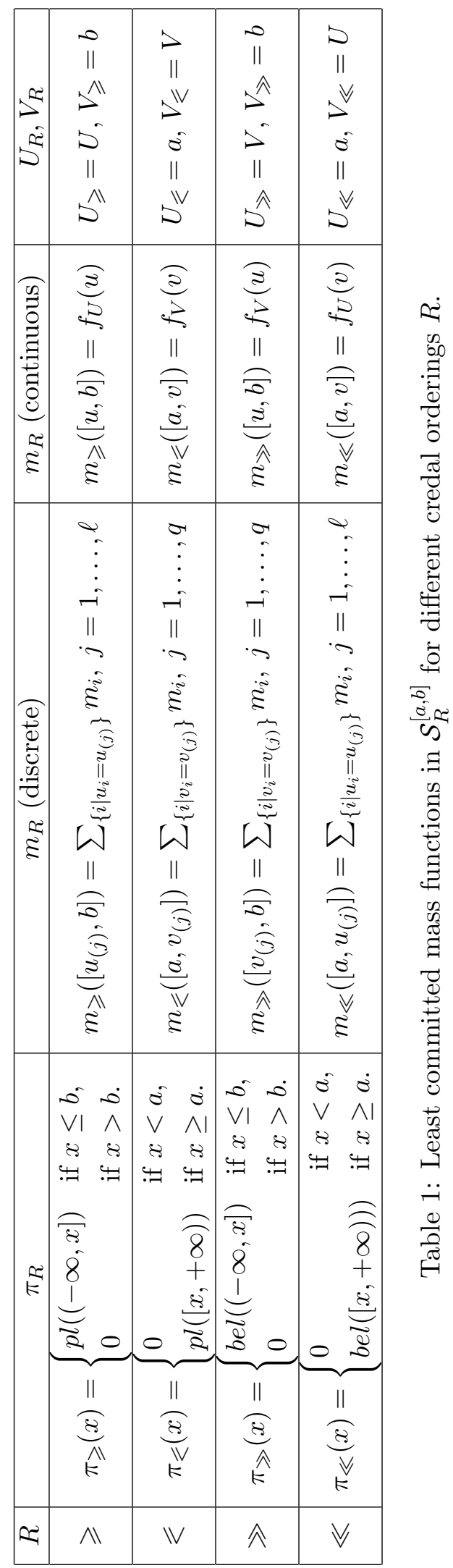

